\title{
Ergonomics and design in a ischial support proposal for the Medellin metro, Colombia
}

\author{
Luz Mercedes Sáenz Zapata ${ }^{1}$ and Andrés Valencia Escobar ${ }^{2}$ \\ ${ }^{a}$ Ergonomics Research Division, Design Studies Research Group, Industrial Design Faculty, Universidad \\ Pontificia Bolivariana, Circular 1 \# 70-01 Bloque 10, Medellín, Colombia. \\ ${ }^{\mathrm{b}}$ Experimental Morphology Research Division, Design Studies Research Group Industrial Design Faculty, \\ Universidad Pontificia Bolivariana, Circular 1 \# 70-01 Bloque 10, Medellín, Colombia.
}

\begin{abstract}
This project, developed by the Design Studies Research Group at the Universidad Pontificia Bolivariana (UPB), presents a design proposal focused on human factors for ischial support for the stations of the Medellín Metro. An initial pilot scheme was developed on Platform 'A' of San Antonio - the most important transfer station. The methodology of the Ergonomics Research Division at UPB constituted the basis of the project. This methodology observes, analyses and draws conclusions of the conditions that optimize the User-Product-Context nexus, and in doing so defines design requirements which, in this proposal, centered on the needs of the users of the Medellín Metro. The Experimental Morphology Research Division provided technical support in the form of modelling, production and structural testing of the object. The proposal includes thematic units, themes and priority components for analysis and application in the design process. In addition, the project generates certain activities and moments that future designers can develop in a parallel manner, coherent with the vision of ergonomics. The methodology focused on the requirements of the users and took into account the existing space (the Metro System), thus establishing relationships of use that were coherent with the principles of ergonomics and design.
\end{abstract}

Keywords: street furniture, reduced mobility, user centered

\section{Introduction}

This paper presents a design proposal for an ischial support for the stations of the Medellín Metro system. The project was carried out by the Design Studies Research Group at the Universidad Pontificia Bolivariana(UPB). An initial pilot scheme was developed on platform ' $A$ ' of San Antonio station. The objectives of this project were: to identify the support needs of people with reduced mobility; to identify situations in which physical support would be necessary; to define the requirements for the design proposal; to design the proposal; to develop and install two prototypes (one on either side of the platform); carry out technical and user tests to determine the final design of the support.

\footnotetext{
${ }^{1}$ E-mail: 1uzmercedes.saenz@upb.edu.co

${ }^{2}$ E-mail: andres.valencia@upb.edu.co
}

The methodology of the Ergonomics Research Division at UPB [8] constituted the basis of the project. This methodology observes, analyses and draws conclusions about the conditions that optimize the UserProduct-Context nexus, and in doing so defines design requirements which in this proposal centered on the needs of the users of the Medellín Metro. The Experimental Morphology Research Division provided technical support in the form of modelling, production and structural testing of the object.

\section{Background}

Since 2003, the Medellín Metro has introduced a number of upgrades that have enabled the system to be more easily accessed by people with reduced mo- 
bility. Upgrades, which include the installation of lifts, ramps and electromechanical platforms, have been installed in thirty of the system's thirty-one stations [6].

The ability to move freely in the city and enjoy the city's transport systems is contingent on a number of factors, including the well-being of the individual: physical disability, visual and/or hearing impairment, infirmity and accidents can all hinder an individual's movement. Similar problems can exist for the elderly, expectant mothers and people of short stature. Another factor that can affect an individual's freedom of movement, and their ability to remain in one place for an extended period of time, is the availability of urban furniture for waiting, resting and recuperating.

\section{Framework}

Therefore, in addition to the upgrades that had already been introduced to the system, the Medellín Metro was keen to examine the possibilities of incorporating ischial support into the waiting and seating areas of its stations, thereby greatly increasing the comfort and well-being of those passengers with reduced mobility. These plans were in line with the Metro system's desire to create a culture of inclusiveness.

Place the file in any of the directories where MS Word looks for templates. These directories are defined within MS Word under Tools/Options/File Locations.

\subsection{Reducing mobility}

According to Colombian Law 1287 (2009), reduced mobility is defined as "a restriction in mobility due to any physical disability, or a limitation in a person's ability to interact with their environment..." [4].

Essentially, people with reduced mobility:

- Can have difficulty moving

- Can have difficulty remaining in one place for an extended period of time.

- Can have difficulty standing up from a sitting position.

- May require the use of one or both hands to grasp a walking aid.

- Can suffer reduced mobility due to weight issues.
- Can have difficulty accessing or reaching due to short stature or other anthropometric characteristics.

- Require the removal of architectural barriers to facilitate access and movement.

\subsection{Ischial support}

Ischial support is a component of 'street furniture'. It is installed horizontally and provides support to the body by allowing the user to adopt a semi-sedentary posture. This definition is included in Colombian Law: Decree 1660 - Ch.2 (2003) regulates transport accessibility for the general population, and in particular those with mobility disabilities [5].

Its height and configuration is designed to provide support for short periods of respite. It is designed to benefit everyone; however the main objective is to provide a system of support for those people with reduced mobility.

\subsection{User-Centered Design}

User-Centered Design (UCD) is a methodological tool that aids the design process. Following on from Norman [7], a design process supported by UCD should:

- Make it easy to determine what actions are possible at any moment (make use of constraints).

- Make things visible, including the conceptual model of the system, the alternative actions, and the results of actions.

- Make it easy to evaluate the current state of the system.

- Follow natural mappings between intentions and the required actions; between actions and the resulting effect; and between the information that is visible and the interpretation of the system state.

\subsection{Ergonomics}

"Ergonomics (or human factors) is the scientific discipline concerned with the understanding of the interactions among humans and other elements of a system, and the profession that applies theoretical principles, data and methods to design in order to optimize human wellbeing and overall system performance.

Practitioners of ergonomics, ergonomists, contribute to the planning, design and evaluation of tasks, jobs, products, organizations, environments and sys- 
tems in order to make them compatible with the needs, abilities and limitations of people"[2].

\subsection{Usability}

'Usability' pertains to how easy a product can be used. The ISO (International Standards Organization) defines it as "...the effectiveness, efficiency and satisfaction with which specified users can achieve specified goals in particular environments (ISO DIS 9241-11) [3].

'Usability' and 'ergonomics' are compatible because they share a common goal - the generation of optimum conditions for users.

It does not necessarily follow that a product that is usable for one person will be usable for another. However, objects do possess a number of characteristics that can indicate usability [3].

\section{Methodology}

Development of the project began with a methodological proposal by the Ergonomics Research Division (GED) of the Design Studies Research Group. Their vision of ergonomics is anthropocentric (the starting point is the individual and subsequent conditions of well-being, health and security), systemic (they observe, analyze and draw conclusions about the conditions that optimize the User-ProductContext nexus) and interdisciplinary (the support of other disciplines leads to a more integrated vision of the situation under analysis).

From a starting point of the User-Product-Context nexus, the proposal includes thematic units, themes and priority components for analysis and application in the design process. In addition, the proposal generates certain activities and 'moments' that future designers can develop in a parallel manner, coherent with the vision of ergonomics [8].

The methodology focused on the requirements of the users and took into account the existing space (the Metro System), thus establishing relationships of use that were coherent with the principles of ergonomics and User-Centered Design.

Table 1.showsthe specific variables that were considered in order to obtain information, and indicators for monitoring the users in their assessment tests. These were subsequently used to help define the design requirements, in line with the existing space of the Metro stations and observation of user habits whilst they were waiting on the platform.
Table 1

Thematic units and variables for analysis

\begin{tabular}{|c|c|c|}
\hline Variables & \multicolumn{2}{|c|}{ Indicators } \\
\hline \multirow[t]{3}{*}{ User } & $\begin{array}{l}\text { Reduced Mobility } \\
\text { Person }\end{array}$ & \multirow{8}{*}{$\begin{array}{ll}- & \text { Precedents } \\
- & \text { Legislations and } \\
& \text { Regulations } \\
- & \text { Framework } \\
- & \text { Analysis and } \\
& \text { diagnosis actual } \\
& \text { situation } \\
- & \text { Opportunities and } \\
& \text { requirements of } \\
\text { design. }\end{array}$} \\
\hline & Platform habits & \\
\hline & $\begin{array}{l}\text { Measurements } \\
\text { relationships }\end{array}$ & \\
\hline Product & $\begin{array}{l}\text { Material and form } \\
\text { criteria }\end{array}$ & \\
\hline \multirow[t]{4}{*}{ Context } & San Antonio station & \\
\hline & Accesses & \\
\hline & $\begin{array}{l}\text { Circulation- } \\
\text { Movements }\end{array}$ & \\
\hline & Stay & \\
\hline
\end{tabular}

The project was developed in five phases. Each phase comprised a series of activities:

\subsection{Phase I. Information}

This was the first moment/activity in which points of interest were identified, namely the characteristics discernible in the User-Product-Context thematic units. This provided a clear understanding of the capabilities and limitations of the user, their psychophysical requirements and cognitive features, their behavior and their routines. This phase identified physical and social dimensions, and the characterization of the product and the context of its use (attributes which define the degree of suitability to users and their levels of well-being, health and security).

The activities in phase 1 were:

- Documentation: research on existing ischial supports, typologies, antecedents, legislation and standards in the area of reduced mobility and the Metro system.

- Study of the user and the context: observation and recording of user types (age range, gender and mobility), platform habits, types of movement in the stations, use of existing seating arrangements.

- Diagnosis and analysis of: the situation of disability in the region, existing seating availability in the Metro system, the dynamics of movement in the station, existing conditions of accessibility.

- Conceptualization: definition of concepts that would explain the project's values: the functional (its use); the communicative (what it means and what it expresses); criteria related to production; considerations relating to the philosophy of the Medellín Metro and their intention of offering a transport system available to everybody. 
- Definition of requirements: definition of the criteria and elements for the characterization of form, material and appearance of the ischial support. Criteria and elements were expressed as part of the components of the design process, as seen in the pedagogic model of the Faculty of Industrial Design: The Functional-Operative component (technical features and the ManObject relationship), the AestheticCommunicative component (the appearance and aesthetic qualities of the product) and the Techno-Productive component (the production and maintenance of the product).

The dimensional relationship i.e. the relationship between human dimensions (anthropometric information), and the dimensions of the object, were seen as determinate indicators of the product in order to make it functionally efficient, effective and satisfactory.

\subsection{Phase II. Formalization}

Once information had been processed, and the analysis model had been applied, characteristics were defined that would give form to the object. The requirements that resulted from the first phase were integrated and applied in a manner that responded to the requirements of the user, and consistent with the context of use. The following activities were developed:

- Outlining of design proposals.

- $\quad$ Selection of proposals.

\subsection{Phase III. Materialization}

Prototyping and modelling: Initial characteristics, such as shape and form, were established through the development of a series of basic prototypes, see figure 2 to figure 4 . In order to collect information from the first prototype, a digital model of ischial support was developed using the program Solid Edge. Once a final design had been agreed, a working prototype was installed in the station.

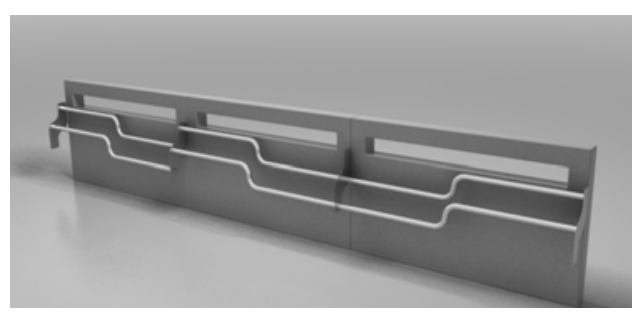

Figure 1. Modelling Object

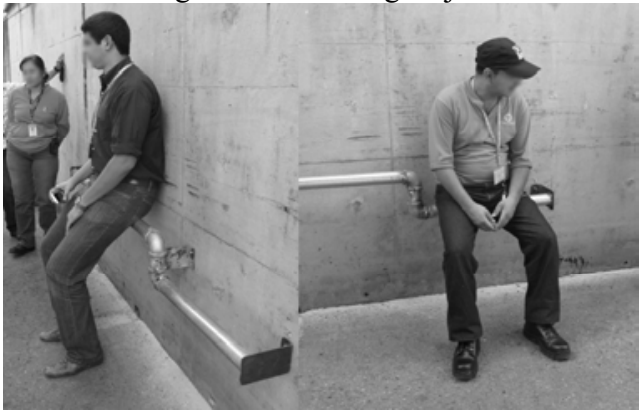

Figure 3. Basic prototype

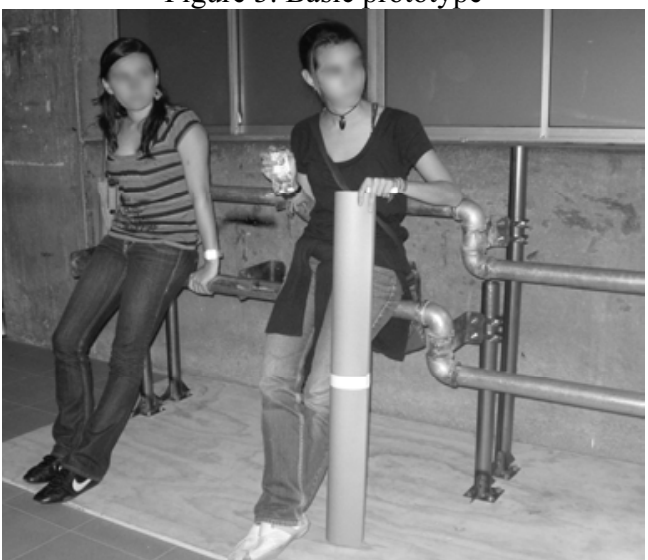

Figure 4. Basic prototype

\subsection{Phase IV. Evaluation}

Functional and operational tests: two groups of tests were carried out. Technical tests were conducted to ensure the structural safety of the object; these tests guaranteed that the structural design criteria (strength, firmness and stability) met the correct standards, and ensured that the mechanical performance of the system ran at an optimum level [9].

Technical tests were also conducted that measured the static structural safety factor of the prototypes. These tests analyzed the tension and distortions of each critical point in the structure and were verified by the Von Misses failure criteria [9]. Production feasibility was constantly monitored by ensuring both the attainability of raw materials in the correct shapes 
and sizes, and that the manufacturing techniques of forming, joining and finishing were achievable.

Once the digital model had been optimized, a functional prototype was installed on the platform for a test period of one month. The information was gathered from this prototype (figure 5) was used to make adjustments to the design. Final specifications based on materials, dimensions and installation/operational conditions were then forwarded to the client.

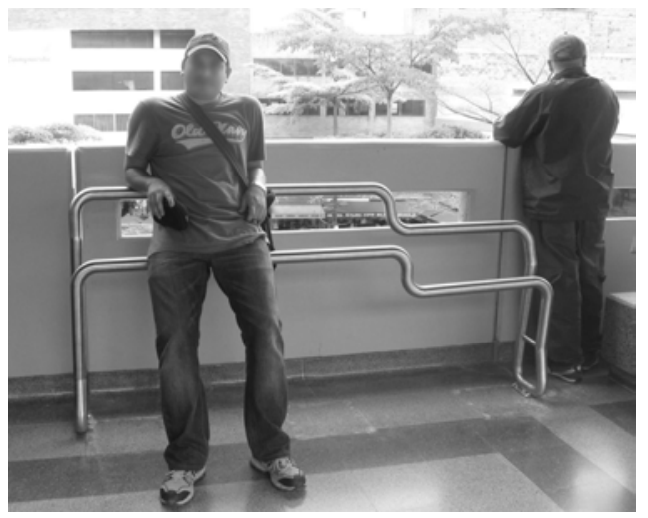

Figure 5. Prototype for user test

The objective of the second set of tests was to evaluate the relationship of use with the prototypes (conditions of usability). Tests using the basic prototype determined the height of the ischial support, taking into account people of normal height and people of short stature. A functional prototype was produced, and further evaluations were conducted. These evaluations involved observing a diverse range of passengers - male and female, young and old, people with and without reduced mobility - as they came into contact with the product which had been installed on the eastern platform of Line A at San Antonio station. These tests were conducted over a period of twentysix days. Final adjustments were made, and a further five prototypes were produced as a pilot batch (figures 6 and 7).

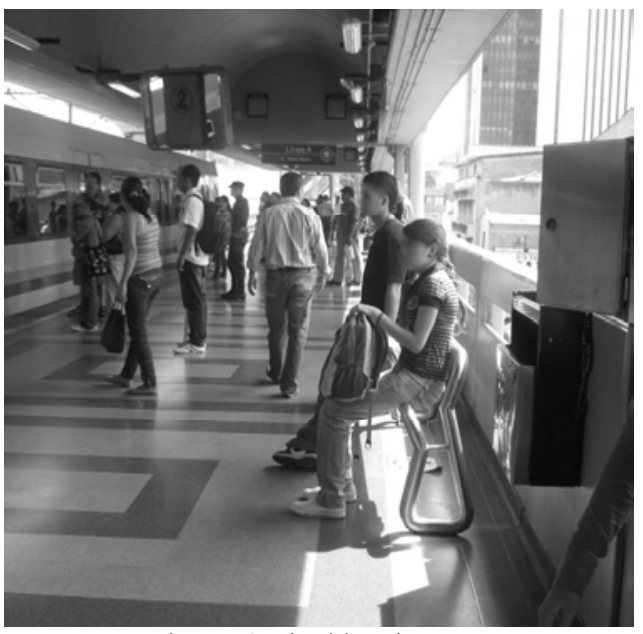

Figure 6. Final batch prototype

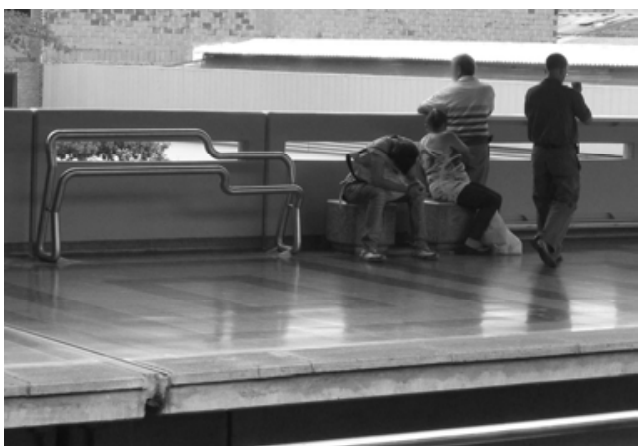

Figure 7. Prototype installed in Metro system station

\subsection{Phase IV. Detail}

- Design specifications.

- Design plans.

- Design report

Shown below are the definitive characteristics of the product, expressed in the Faculty of Industrial Design's Pedagogic Model: Functional -Operative, Techno - Productive, Aesthetic - Communicative [1].

Criteria of the Functional - Operative component (technical features, features relating to usefulness and in the Human - Object relationship):

- The support allows the users to adopt a semi-sitting posture that distributes the weight exerted upon the feet. This in turn generates partial but effective support for the hip area, resulting in a period of rest for the user.

The support provides support for two areas of the body: the hip area and the back/elbows.

The dimensions and texture of the support's interfaces guarantee the avoidance of discomfort and pain. 
Two levels, located at different heights, means that the support can be utilized by people of varying statures. The Inclusive Design approach takes into account not only those people with reduced mobility but also those of short stature.

The support can be used by three adults, in a stationary position, each weighing no more than 85 kilos: two positioned at the upper height; one at the lower.

The support is $100 \%$ resistant to oxidization and suited to outdoor use.

The support requires minimal maintenance; once a month it is necessary to check the tightness of the anchor bolts.

In regular conditions of use, the support is safe and secure for the user; all edges have been smoothed and all joins are hidden.

Criteria of the Techno - Productive component (production and maintenance of the product):

- The support is morphologically formed from one continuous line of pipe.

The entire support is made from just one material, thus optimizing the production process.

The anchorage system used allows the support to be quickly dismantled if necessary.

Criteria of the Aesthetic - Communicative component (appearance and aesthetics of the product):

- The standard polished stainless steel finish lends the object a tone of sobriety.

The communicative function makes the object instantly inviting to the user; they have an almost intuitive understanding with respect to its utilization.

The object's formal configuration and finishing guarantees consistency with other objects and structures found in the station.

\section{Results}

Five final version prototypes were produced and installed in different Metro stations, all of which benefited from high footfalls. In accordance with the wishes of the Medellín Metro, the universal symbol of disability was displayed beneath the ischial supports to indicate clearly that those with reduced mobility had priority in their use.

After a period of four months, the Medellín Metro reported satisfaction amongst users. There were, however, some who requested that the height of the support be decreased.

The Ergonomics Research Division at UPB responded by carrying out further tests with users. Rec- ommendations were forwarded to the Medellín Metro; these recommendations would be taken into account when installing ischial supports in other Metro stations.

Recommendations put forward to the Medellín Metro centered on:

Safeguarding the dimensions of the ischial support; the need to create a culture based on the themes of the project (reduced mobility, ischial support, semisedentary posture); a clearer, more effective system of visuals in order to facilitate the use of the support; the initiation of a campaign to increase understanding of how the support works and the users that have priority, based on the Metro's experience in creating a culture of inclusiveness: For Everybody.

\section{Conclusions}

This experiment confirms that the relationship between universities and industry - within a framework of research and development, whose shared objective is the furtherance of human well-being - can result in more creative and productive work environments.

The design process runs parallel with, and is complementary to, the objectives of ergonomics: both observe and evaluate the users, their characteristics and their requirements.

Student participation in research training - their contact with users in varying contexts - suggests that these experiments constitute an alternative way of learning.

In total, thirty ischial supports have been installed in high-footfall stations of the Medellín Metro. This project has reaffirmed the Medellín Metro's commitment to social responsibility and inclusiveness in Medellín and the wider metropolitan area.

\section{Acknowledgements}

The authors wish to thank all the members of the work team for their assistance in the development of project activities: Camilo Andrés Páramo V., Industrial Designer and teacher in Industrial Design Faculty(UPB), Juan Esteban Vélez V., Mechanical Engineer (UPB), Juan Sebastián García C., student in Industrial Design Faculty(UPB), Liliana Sanín S. coordinator in University-Industry Projects Office in Industrial Design Faculty(UPB) and Adriana Arcila and partners in Metro de Medellín Planning Office. 


\section{References}

[1] Facultad de Diseño Industrial, Escuela de Arquitectura y Diseño. Medellín, 2009. Modelo disciplinar de la facultad de diseño industrial. Universidad Pontificia Bolivariana. Medellín, Colombia.

[2] International Ergonomics Association. What is Ergonomics. Accessed IEA Council, 2000. [Accessed August 19, 2011] httphttp://www.iea.cc/01_what/What\%20is\%20Ergonomics.ht $\mathrm{ml}$

[3] Jordan, Patrick. An Introduction to Usability. United Kingdom: 1998. P.5 ISO (International Standard Organization) Concepto de Usabilidad. 1998. p.5

[4] Ley 1287 de 2009, Artículo 5. Bogotá: Presidencia de la República; 3 de Marzo de 2009 [AccessedAugust 19, 2011] 011]. Archivo Leyes 2009.web.presidencia.gov.co/leyes/2009/marzo/ley128703032 009.pdf

[5] Ley 1660 de 2003. Bogotá: Presidencia de la República Ministerio de Transporte; 16 de Junio de 2003 [Accessed: September30th, 2010]. Decretos en Línea. http://www.presidencia.gov.co/prensa_new/decretoslinea/

[6] Metro de Medellín. Accesibilidad para Personas con Movilidad Reducida -PMR-.Medellín: Empresa de Transporte Masivo del Valle de Aburrá Limitada; [Accessed: August 19, 2011].

http://www.metrodemedellin.gov.co/index.php?option=com_c ontent\&view $=$ article $\&$ id $=62 \&$ lang $=$ es

[7] Norman, Donald. La psicología de los objetos cotidianos Madrid, Editorial Nerea. 1990. p. 231-265.

[8] Sáenz, Luz Mercedes. En el proceso de diseño: alternativa metodológica para la concepción de productos. Medellín, editorial Universidad Pontificia Bolivariana. Revista Iconofacto 2008; 4(5):170-182.

[9] Valencia, Andrés Hernando. La estructura, un elemento técnico para el diseño. Medellín, Editorial Universidad Pontificia Bolivariana. 2007 p.265-308 